\title{
Azotemia in Gastro-intestinal Haemorrhages
}

The problem of kidney impairment after severe haemorrhage is one of great importance. Oliguria, or complete suppression of urine commonly follows a prolonged period of renal anoxia due to anaemia and low blood pressure, and this may occur after gastro-intestinal

\section{Editorial}

\section{1}

haemorrhage just as after abortions. In this condition the non-protein nitrogen in the blood may rise to extreme heights and the electrolyte balance in the body need expert and constant control. In less severe degrees of haemorrhage as from a peptic ulcer, it is usual to find a raised blood urea, and this is often used as a rough guide to the degree of dehydration. In a recent study of 27 patients carried out be $\mathrm{H}$. N. Haugen *, however, the author points out that the increase in blood urea has other causes than renal impairment. As he makes clear, the main source of the urea in the blood is normally the protein taken as food, and the amount of protein in a litre of blood is approximately 100 grams. From this it follows that blood passed into the alimentary tract will tend to cause a rise in blood urea when it is digested and absorbed. Creatinine, on the other hand, in digested blood is without importance, as a litre of blood contains less than 0.2 grams of this substance. For this reason parallel studies of urea and creatinine in the blood of patients after haematemesis or melaena should throw some light on the degree of renal impairment for, if this was severe, the creatinine must rise in the blood, which it would not do merely from the absorption of digested blood.

Amongst the patients studied this relationship appeared to hold true, though the author could not, of course, be sure how much blood in any individual case was actually lost into the intestine. In cases showing only moderate anaemia and no appreciable shock, creatinine values in the blood were all normal. In those in whom exhaustion, low blood pressure, shock and marked anaemia occurred, both blood urea and blood creatinine values were raised, as compared to the elevation only of the blood urea in the less severe cases.

It seems clear that after gastro-intestinal haemorrhage, the increase in blood urea is mainly due to intestinal digestion and resorption of blood and that only in more severe cases with low blood pressure and shock, is depression of the renal function an additional cause. This depression, the author concludes, can most easily be diagnosed by the demonstration of an increased plasma creatinine concen tration. Th. H.

- Haugen, H. N.: Scand. J. Clia. and Lab. Invest. 1955, Vol. 7, No. 1, p. 75. 